\title{
Optimal Sizing of ESS for Reducing AGC Payment in a Power System with High PV Penetration
}

Zhang, F.; Fu, A.; Ding, L.; Wu, Qiuwei; Liang, Z.

Published in:

International Journal of Electrical Power \& Energy Systems

Link to article, DOI:

10.1016/j.ijepes.2019.03.029

Publication date:

2019

Document Version

Peer reviewed version

Link back to DTU Orbit

Citation (APA):

Zhang, F., Fu, A., Ding, L., Wu, Q., \& Liang, Z. (2019). Optimal Sizing of ESS for Reducing AGC Payment in a Power System with High PV Penetration. International Journal of Electrical Power \& Energy Systems, 110, 809818. https://doi.org/10.1016/j.ijepes.2019.03.029

\section{General rights}

Copyright and moral rights for the publications made accessible in the public portal are retained by the authors and/or other copyright owners and it is a condition of accessing publications that users recognise and abide by the legal requirements associated with these rights.

- Users may download and print one copy of any publication from the public portal for the purpose of private study or research.

- You may not further distribute the material or use it for any profit-making activity or commercial gain

- You may freely distribute the URL identifying the publication in the public portal 


\title{
Optimal Sizing of ESS for Reducing AGC
}

\section{Payment in a Power System with High PV}

\section{Penetration}

\author{
F. Zhang ${ }^{\mathrm{a}}$, A. Fu ${ }^{\mathrm{b}}$, L. Ding*a, Q. Wu ${ }^{\mathrm{c}, \mathrm{d}}$, Z. Liang \\ ${ }^{a}$ Key Laboratory of Power System Intelligent Dispatch and Control of Ministry of Education, Shandong \\ University, No.17923, Jingshi Road, Ji'nan 250061, China, \\ ${ }^{b}$ Department of Electrical Sustainable Energy, Delft University of Technology, 2628, CD, Delft, Netherlands, \\ ${ }^{c}$ Center for Electric Power and Energy (CEE), Department of Electrical Engineering, Technical University of \\ Denmark (DTU), 2800, Kgs. Lyngby, Denmark, \\ ${ }^{d}$ School of Engineering and Applied Sciences, Harvard University, 29 Oxford Street, Cambridge, MA, 02138, \\ USA, \\ e Institute for Manufacturing, University of Cambridge, 17 Charles Babbage Rd, Cambridge, CB3 OFS, UK
}

Corresponding author: Tel.: +86 531-81696119. E-mail address: dinglei@sdu.edu.cn

\begin{abstract}
Rapid and large variation of photovoltaic (PV) power may incur frequency variation in a power system with high PV penetration. In such a case, much more reserve capacity of automatic generation control (AGC) for frequency regulation will be needed. In this paper, the energy storage station (ESS) is used to decrease the ramp rate of the total PV power and consequently decrease the AGC reserve capacity requirement of the power grid. However, as an expensive option, the ESS capacity should be optimally determined considering the balance between ESS cost and the equivalent benefit from the reduction of AGC payment. Therefore, an optimal sizing scheme for ESS in a power system with high PV penetration is proposed to strike a balance between the ESS cost and the reduction of the AGC payment. Firstly, a control scheme of ESS is proposed for smoothing PV power variation, during which the ramp rate of PV power can be finitely controlled with a size-limited ESS. Afterwards, an object function aiming to achieve the tradeoff between the investment cost of ESS and the reduction of AGC payment for frequency regulation is presented. Finally, case studies are conducted with actual PV power data to verify the effectiveness of the presented sizing scheme.
\end{abstract}

Keywords: energy storage; optimal sizing; photovoltaic power; ramp rate

\section{Introduction}

Due to the environmental concern and quest of energy supply security, the scale and penetration of renewable energy is increasing rapidly. In contrast with wind power, photovoltaic (PV) power can drop much faster, e.g., as high as $60 \%$ of the installed power capacity per minute when cloud passes [1]. In such a case, in a power system with high PV penetration, the PV power variation may incur frequency deviation, and much more reserve capacity of automatic generation control (AGC) will be required for frequency regulation. 
Therefore, counter-measures should be developed to mitigate the rapid and large power variation in a power system with high PV penetration, which is a serious threat to the secure operation of power systems [2].

Normally, thermal generators are responsible for frequency regulation of power systems [3]. However, due to the limited ramp rate and reserve capacity of thermal generators, in power systems with high PV penetration, frequency deviation cannot be effectively handled. Consequently, due to the fast power response ability, the technology of energy storage can be used to mitigate the PV power variation and equivalently improve the frequency stability [4], [5].

In the existing literatures, normally the dispersed energy storage unit (ESU) is located in PV stations, when the ESU is used to smooth PV power output. A new approach for ramp rate control of $\mathrm{PV}$ power was developed to mitigate the power variation caused by cloud passing with the ESU in [1]. A real-time coordinated control scheme was proposed in [6] for voltage regulation in weak distribution systems with high PV penetration, in which charging/discharging power of the storage system was adaptively tuned by an active adjustment method in order to avoid battery premature energy exhaustion. In [7], a state of charge (SOC) feedback battery control strategy was presented, and a real-time power allocation method for power and energy regulation of ESUs was proposed, in which the rate of PV power fluctuation and the allowable maximum charge/discharge power and energy of each battery unit were considered. In [8], the ramp rate control of a PV generator with an electric double-layer capacitor was presented, and after smoothing the PV power variation, the ramp rate of the PV power could be limited. In [9], a new method for a gridconnected PV storage system was proposed to shift energy for arbitrage and power smoothing with two in-dependent storage units. A model predictive control (MPC) based control strategy for PV plants was presented in [10] with the ESU to be scheduled in the daily and intraday electricity market.

On the other hand, the energy storage is still an expensive option currently. Therefore, optimally determining the energy storage capacity needs to be solved [11]. In [12], the size of the battery ESU on a university campus was optimally determined with the prediction of load and PV generation. A new battery sizing method for the grid-connected PV plant application was proposed in [13], considering the technical requirement and economic benefits of power systems. An optimization model for sizing battery storage of gridconnected PV systems was presented in [14], in which the objective was to minimize the cost associated with net power purchase from the electric grid and the battery capacity loss considering the electricity price change. In [15], a performance-based sizing method was presented for ESU at the house, distribution transformer, and community levels with high solar PV penetration. In [16], a new method to select the optimal size of superconducting magnetic energy storage was proposed by using the simplex optimization algorithm. Several factors, such as the output power fluctuation, the efficiency of converter, and voltage regulation were considered during the optimization process.

From the above research, it can be seen that the dispersed ESUs are normally located in 
distributed PV power plants, and the purpose of ESU utilization is to smooth the power variation and/or optimize the investment cost for PV station owners. In fact, in a power system with high PV penetration, the PV power fluctuation significantly increases the automatic generation control (AGC) reserve capacity and the operation cost of the power system as well. Hence, from the operation perspective of the power system, an independent energy storage station (ESS) can be adopted in the power grid. ESS is quite different from the dispersed ESUs, because normally it is installed in an important node of the power grid, rather than the PV plants. Then, considering the natural smoothing effectiveness of PV power located in geographically adjacent areas, ESS can show better performance in PV power fluctuation smoothing, in comparison with the dispersed ESUs. Meanwhile, when determining the ESS capacity, ESS can be considered from the point of view of the whole power gird, such as it will obviously benefit the reduction of AGC reserve capacity. Hence, in contrast with the dispersed ESUs in PV plants, during the sizing procedure of ESS, it is potential for ESS to consider the operation cost of the power gird, e.g., the AGC payment, to generate equivalent benefit from the reduction of AGC payment.

For this reason, an optimal sizing scheme for ESS considering the AGC payment in a power system with high PV penetration is proposed in this paper. Firstly, the calculation of the AGC reserve capacity requirement is introduced, which is the main factor of AGC payment. Meanwhile, the influence of PV variation on the AGC reserve capacity is analyzed in detail. Afterwards, a new control strategy for ESS is presented aiming to effectively control the ramp rate of PV power with the size-limited ESS. Correspondingly the AGC reserve capacity requirement with ESS integrated is also be analyzed. Subsequently, to achieve a tradeoff between the reduction of AGC payment and ESS investment cost, a cost-benefit model for the ESS is designed to determine the optimal ESS capacity. Using the actual PV power data, case studies are conducted to verify the effectiveness of the proposed sizing scheme.

The rest of this paper is organized as follows. The determination of the AGC reserve capacity requirement in a power system with high PV penetration is introduced in Section II; the control strategy of ESS for limiting ramp rate of PV power is described in Section III; the ESS sizing scheme is presented in Section IV; case studies are given in Section VI; and, finally, the conclusion is drawn in Section VII.

\section{Analysis of AGC reserve capacity requirement}

\subsection{Calculation of $A G C$ reserve capacity}

The cost of AGC reserve capacity is a main factor of AGC payment in a power gird. Normally, AGC mainly responds to the minute to minute load variation. Considering the feature of PV power, it can be observed that the variation of PV power is corresponding to the time scale of AGC, and then in a high PV penetration grid, when large ramp rate of PV power occurs, much more AGC reserve capacity will be needed. Normally the curve of AGC reserve capacity is a staircase function with 24 equal line segments, implying the AGC reserve capacity is hourly determined. When considered in the calculation of AGC 
reserve capacity, PV power can be treated as a kind of negative load, which is expressed as follows.

$$
P_{e l}(t)=P_{l d}(t)-P_{p v}(t)
$$

where $P_{e l}(t)$ is the equivalent load. $P_{l d}(t)$ is the actual load. $P_{p v}(t)$ is the PV power. Accordingly, the minute to minute load variation $P_{e}(t)$ can be determined as

$$
P_{e}(t)=P_{e l}(t)-P_{r e f}(t)
$$

where $P_{\text {ref }}(t)$ is the reference value for $P_{e l}(t)$. In this paper, $P_{\text {ref }}(t)$ is determined with moving average method.

$$
P_{r e f}(t)=\frac{1}{M}\left\{P_{e l}[t-(M-1) \Delta t]+P_{e l}[t-(M-2) \Delta t]+\ldots P_{e l}(t)\right\}
$$

where $M$ is the number of the selected sampling points. $M$ is set as 10 in this paper.

Afterwards, the load variation $P_{e}(t)$, which is the power deviation between $P_{e l}(t)$ and $P_{\text {ref }}(t)$, can be determined and used for the calculation of AGC reserve capacity. For example, $P_{e-11}(t)$ is the load variation between 11:00 and 12:00, and then the probability density function (pdf) of $P_{e-11}(t)$ can be calculated. Accordingly, the AGC reserve capacity between 11:00 and 12:00 is determined with the pdf:

$$
F\left[-P_{c-11} \leq P_{e-11}(t) \leq P_{c-11}\right]=p
$$

where $F$ is the pdf. $P_{c-11}$ is the AGC reserve capacity between 11:00 and 12:00. When $p$ is $0.998, P_{c-11}$ can be determined as $11.7 \mathrm{MW}$, as shown in Fig.1. Accordingly, the houly AGC reserve capacity of one whole day can be determined using the pdf of $P_{e}(t)$.

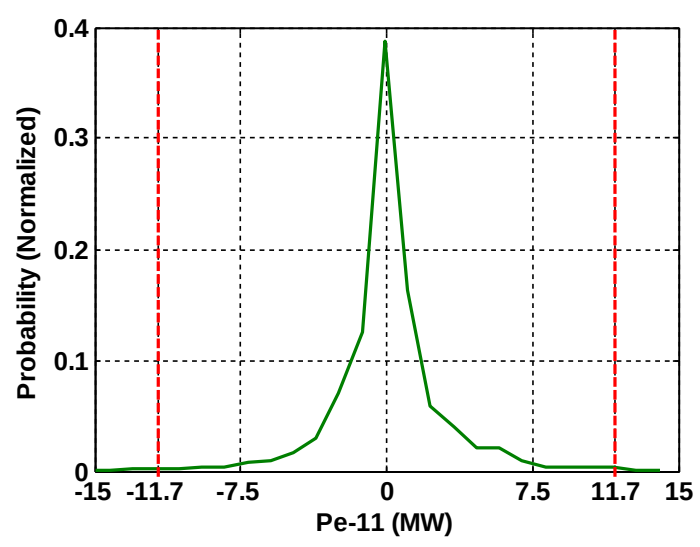

Fig. 1. pdf of $P_{e-1 l}(t)$

\subsection{Influence of $P V$ power variation on $A G C$ reserve capacity requirement}

When cloud passes, the ramp rate of PV power shows various features. Generally four typical scenarios can be used to show the detailed features, as shown in Fig.2. In the first scenario in Fig.2 (a), the sharp power decrement during $\left[t_{1}, t_{2}\right]$ and sharp power increment during $\left[t_{3}, t_{4}\right]$, which have large downwards and upwards ramp rate respectively, are included, and meanwhile the PV power keeps constant during $\left[t_{2}, t_{3}\right]$ for a short while. Scenario (a) can reflect the phenomenon when large cloud is passing. During $\left[t_{1}, t_{2}\right]$, the 
large cloud gradually covers the PV plant, and the PV power varies with a relatively high ramp rate. Afterwards, during $\left[t_{2}, t_{3}\right]$ the cloud totally covers the PV plant and the PV power keeps constant with a lower value for a while. During $\left[t_{3}, t_{4}\right]$, the cloud moves away and incurs the increment of PV power with a relatively high upward ramp rate. Subsequently, in scenario (b), the PV power sharply decreases during $\left[t_{1}, t_{2}\right]$, and then immediately the power increases with a high ramp rate during $\left[t_{2}, t_{3}\right]$. Scenario (b) is responding to the phenomenon when a relatively small piece of cloud is quickly passing. Similarly, in scenario (c), the power decreases mildly during $\left[t_{1}, t_{2}\right]$, and then the power sharply increases immediately during $\left[t_{2}, t_{3}\right]$. In the fourth scenario, the power decreases sharply during $\left[t_{1}, t_{2}\right]$, and then the power mildly increases immediately during $\left[t_{2}, t_{3}\right]$. The difference between scenario (c) and (d) is mainly related with the cloud moving speed. Generally, the four scenarios can be referred to various natural phenomenons.

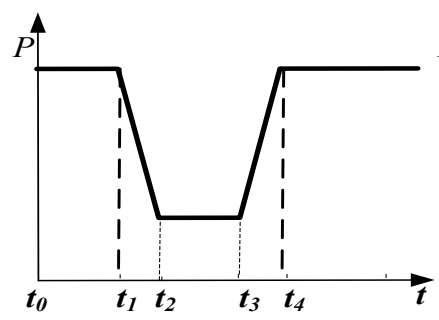

(a)

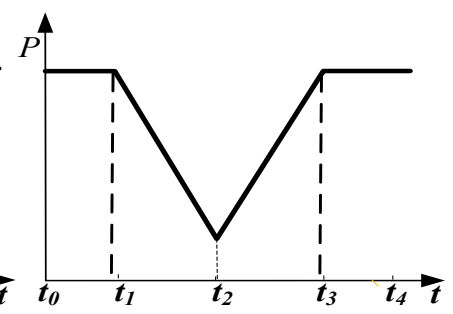

(b)

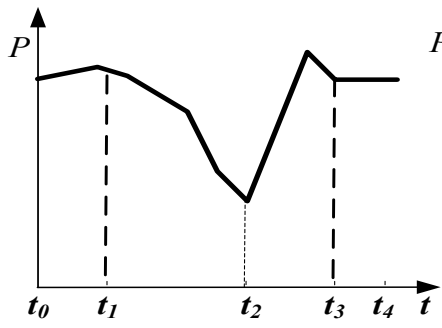

(c)

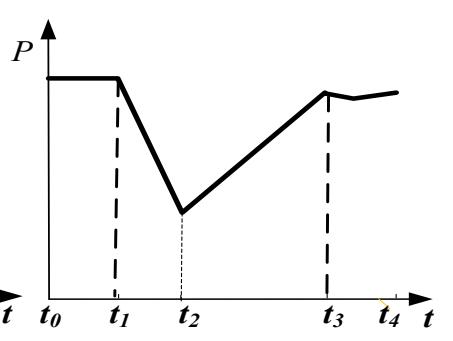

(d)

Fig 2. Scenarios of PV power variation

When the total PV power in the grid is considered in the calculation of AGC reserve capacity, from Eq. (2), it can be deduced that the wild PV power variation can incur obvious load variation. Correspondingly the AGC reserve capacity requirement will be significantly increased. Fig. 3 shows the pdf of load variation during one hour under different PV penetrations. It can be seen that when the PV penetration is increasing, the total PV power variation will be wilder, and then the AGC reserve capacity requirement is becoming higher. Consequently, the PV power variation should be effectively smoothed to decrease AGC reserve capacity. In this paper, considering the limited size of ESS, a novel control strategy of ESS to finitely control the ramp rate of PV power is presented with the aim to decrease the ESS capacity requirement. 


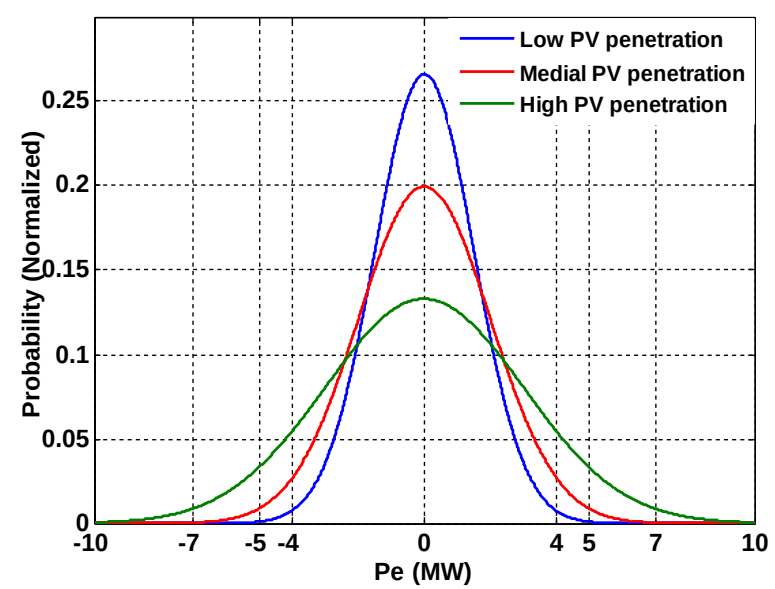

Fig.3 AGC reserve capacities at various PV penetrations

\section{ESS control strategy}

Lithium-ion battery $(\mathrm{LiB})$ has been widely used in engineering projects due to its high energy density and relatively low cost. Even so, nowadays LiB ESS is still an expensive option to smooth the PV power variation. Considering the high investment, the ESS capacity needs to be optimally determined. Accordingly, ramp rate control of PV power will be conducted with a size-limited ESS. However, if the variation of PV power is required to be smoothed perfectly, much higher ESS capacity will be required and more ESS investment will be needed. Hence, finite ramp rate control will be introduced, the advantages of which can be described as follows. On one side, only when the ramp rate of PV power is beyond the setting limit, ESS will start to control the PV power ramp rate to be a reasonable value. Meanwhile, when the ramp rate of PV power variation is within the allowable limit, ESS will not start to decrease the utilization to prolong its lifetime [17]. On the other side, the finite ramp rate control can effectively decrease the variation of PV power to reduce the AGC reserve capacity, and meanwhile it has lower size requirement of ESS in contrast with the case when the PV power variation needs to be perfectly smoothed. For above reasons, the control rules of ESS are presented to finitely control the ramp rate of PV power.

\subsection{Control rules of ESS}

The ramp rate of PV power is defined to show the variations of PV power and the conditions whether ESS will start or not.

$$
\lambda=\frac{\left|P_{p v}(t)-P_{p v}(t-\Delta t)\right|}{\Delta t}
$$

where $\lambda$ is the ramp rate of PV power. $P_{p v}(t), P_{p v}(t-\Delta t)$ are the PV power at $t$ and $t-\Delta t$, respectively. $\Delta t$ is the sampling interval, which is 1 minute in this paper.

Subsequently, ESS will be used to finitely control ramp rate of PV power. When the ramp rate of PV power is larger than the limit value, ESS will start to charge/discharge, and the ramp rate of the smoothed PV power will be constrained within a reasonable range. Specifically, according to the technical rule for photovoltaic power station connected to 
power grid of State Grid Corporation of China, for a medium-sized PV power plant, for example, hundreds of MW, the PV power variation per minute should be less than $20 \%$ of the installed capacity. To strictly constrain the power variation in a power grid with high PV penetration, in this paper, the PV power variation per minute is constrained within $10 \%$ of the installed capacity. Accordingly, when the PV power variation per minute is larger than $10 \%$ of the installed capacity, ESS will charge/discharge to effectively control the ramp rate of $\mathrm{PV}$ power.

Based on the description above, the control rules of ESS can be designed as follows.

(1) ESS will not start when the ramp rate is smaller than the limit value.

As shown in Fig.4, during the time intervals $\left[t_{0}, t_{1}\right]$ in Fig.4 (a)-(d) and $\left[t_{3}, t_{4}\right]$ in Fig.4 (c)-(d), the power variation of PV power is quite small, and

$$
\frac{\left|P_{p v}(t)-P_{p v}^{\prime}(t-\Delta t)\right|}{\Delta t} \leq \lambda_{\text {lim }}
$$

where $\lambda_{\text {lim }}$ is the ramp rate limit of PV power, i.e., $10 \%$ of the installed capacity of PV plant. $P^{\prime} p v(t-\Delta t)$ is the actual PV power output of last moment, i.e., the smoothed PV power at $t-\Delta t$. In such a case, the power deviation between $P_{p v}(t)$ and $P^{\prime} p v(t-\Delta t)$ is within the ramp rate limit, so ESS will not start at $t$. Correspondingly, the smoothed PV power $P$ ' $p v(t)$ can be determined as follows.

$$
P_{p v}^{\prime}(t)=P_{p v}(t)
$$

(2) ESS discharges when the downward ramp rate is larger than the limit value.

During the time intervals $\left[t_{1}, t_{2}\right]$ in Fig.4 (a)-(d), the PV power has downward ramp rate. In such case, the power deviation between $P_{p v}(t)$ and $P^{\prime} p v(t-\Delta t)$ may be beyond the ramp rate limit. If

$$
\frac{\left|P_{p v}(t)-P_{p v}^{\prime}(t-\Delta t)\right|}{\Delta t} \geq \lambda_{\text {lim }}
$$

ESS will discharges to finitely control the ramp rate of PV power. Correspondingly, the smoothed PV power at $t P^{\prime} p v(t)$ can be determined as follows.

$$
P_{p v}^{\prime}(t)=P_{p v}^{\prime}(t-\Delta t)-\lambda_{\lim } \cdot \Delta t
$$

Accordingly, the discharge power of ESS $P_{E d}(t)$ is calculated as

$$
P_{E d}(t)=P^{\prime} \quad p v(t-\Delta t)-\lambda_{\text {lim }} \cdot \Delta t-P_{p v}(t)
$$

(3) ESS charges when the upward ramp rate is larger than the limit value.

During the time intervals $\left[t_{3}, t_{4}\right]$ in Fig.4 (a) and $\left[t_{2}, t_{3}\right]$ in Fig.4 (b)-(d), the PV power has upward ramp rate. If

$$
\frac{\left|P_{p v}(t)-P_{p v}^{\prime}(t-\Delta t)\right|}{\Delta t} \geq \lambda_{\text {lim }}
$$

ESS will charges to finitely control the ramp rate, and the smoothed PV power $P^{\prime} p v(t)$ can be determined as follows.

$$
P_{p v}^{\prime}(t)=P_{p v}^{\prime}(t-\Delta t)+\lambda_{\lim } \cdot \Delta t
$$

Accordingly, the discharge power of ESS $P_{E c}$ can be calculated as 


$$
P_{E c}=P^{\prime} \quad p v(t-\Delta t)+\lambda_{\text {lim }} \cdot \Delta t-P_{p v}(t)
$$

It should be pointed that to distinguish the charge/discharge state of ESS, $P_{E d}$ in Eq. (10) will be positive, and $P_{E c}$ in Eq. (13) will be negative.

According to the control rules of ESS, Fig.4 shows the smoothing process of PV power variation. From Fig.4, it can be seen that in Fig.4 (a), during $\left[t_{0}, t_{l}\right]$ the power deviation between $P_{p v}(t)$ and $P^{\prime} p v(t-\Delta t)$ is within the ramp rate limit, so according to Eq. (7), $P^{\prime}$ $p v(t)$ is equal to $P_{p v}(t)$. Afterwards, during $\left[t_{1}, t_{2}\right]$, a large piece of cloud is passing and the $\mathrm{PV}$ power varies with a relatively large ramp rate which is beyond the ramp rate limit. Consequently, the smoothed PV power $P^{\prime} p v(t)$ will be calculated according to Eq. (9), and ESS will discharge to finitely control the ramp rate of PV power with a specified value in Eq. (10). Specially, during $\left[t_{2}, t_{3}\right]$, although $P_{p v}(t)$ keeps constant, according to Eq. (8), the power deviation between $P_{p v}(t)$ and $P^{\prime} p v(t-\Delta t)$ is still larger than $\lambda_{\text {lim }}$. Accordingly, ESS still discharges for the finite ramp rate control. When the cloud is moving away, during $\left[t_{3}, t_{4}\right]$, the upward ramp rate appears and when the ramp rate is beyond the limit, ESS will charges to finitely control the upward ramp rate, and $P^{\prime} p v(t)$ can be determined according to Eq. (12). Also it should be noted that during $\left[t_{4}, t_{5}\right]$, although the cloud leaves and $P_{p v}(t)$ keeps constant, according to Eq. (11), the power deviation between $P_{p v}(t)$ and $P^{\prime}$ $p v(t-\Delta t)$ is still larger than $\lambda_{\text {lim }}$. Hence, ESS keeps charging according to Eq. (13). In a similar manner, the process of ramp rate control of PV power in Fig.4 (b) - (d) can be analyzed, which are responding to various natural phenomenons.

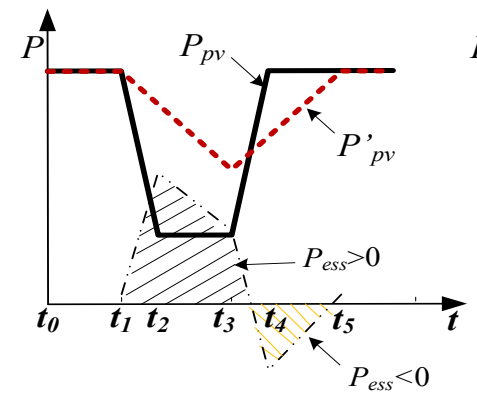

(a)

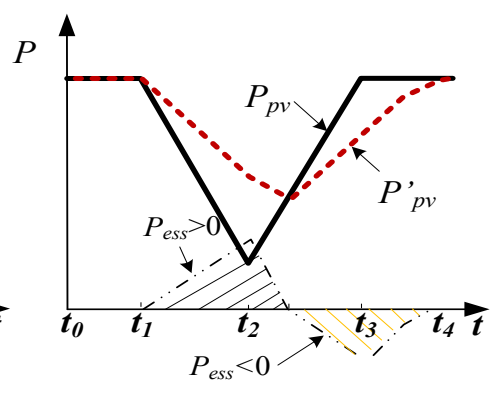

(b)

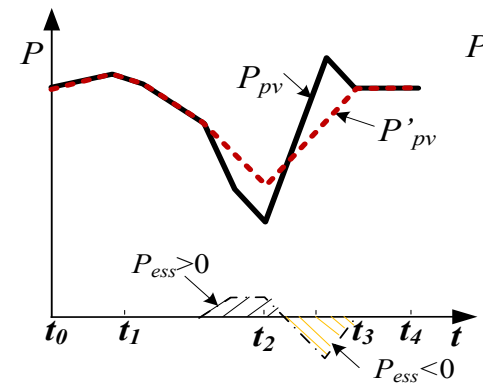

(c)

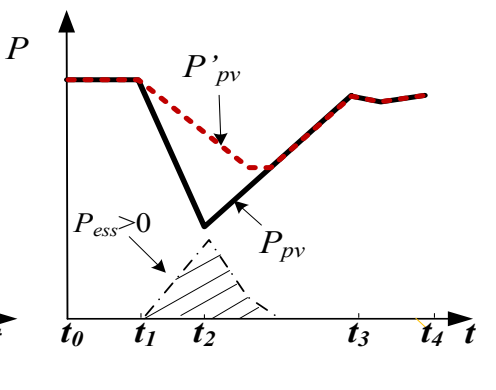

(d)

Fig.4 Finite ramp rate control with ESS

\subsection{Impact of ESS on AGC reserve capacity requirement}


After the ramp rate control of PV power with the presented control rules of ESS, in a power grid with high PV penetration, the AGC reserve capacity requirement can be analysed. According to Eq. (1), when ESS is integrated, the equivalent load can be described as

$$
P_{e l}(t)=P_{l d}(t)-P^{\prime} \quad p v(t)
$$

The power variation of $P^{\prime} p v(t)$ has been mitigated with ESS via the finite ramp rate control. Hence, for a determined load $P_{l d}(t)$, the power variation of the equivalent load, i.e., $P_{e l}(t)$, has also been mitigated. Correspondingly, according to the calculation of $P_{e}(t)$ in Eq.(2), the pdf of $P_{e}(t)$ will be obviously changed. Fig.5 shows the change of the pdf of $P_{e}(t)$. As shown in Fig.5 (b), when ESS is integrated to finitely control the ramp rate of PV power, the AGC reserve capacity of this time interval is $3.4 \mathrm{MW}$. Comparatively, without ESS, according to the determination method of hourly AGC reserve capacity in Eq.(4), the determined AGC reserve capacity is $5.8 \mathrm{MW}$, as shown in Fig.5 (a). Obviously, in a power gird with high PV penetration, when the power variation of PV power has been effectively smoothed, the AGC reserve capacity of the grid can be decreased.

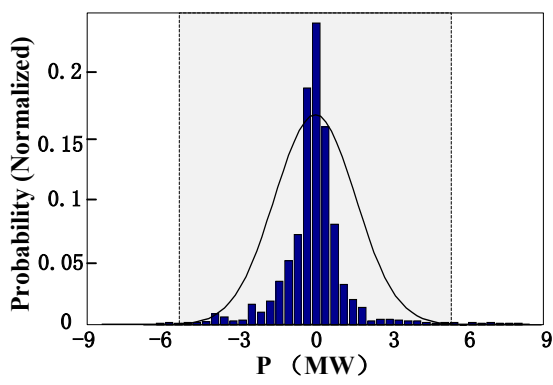

(a)

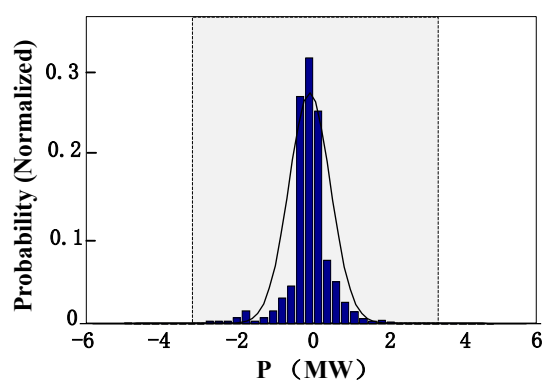

(b)

Fig.5 Comparison of pdf of $P_{e}(t)$ when ESS is integrated or not: (a) without ESS;(b) with ESS

\section{Sizing methodology for ESS considering the cost of ESS and AGC payment}

According to the presented control strategy of ESS, the PV power variation can be effectively smoothed via finite ramp rate control. Subsequently, the ESS size can be optimally determined by analyzing the AGC reserve capacity reduction and the investment cost of ESS, and correspondingly a cost-benefit-based optimization model is presented to minimize the sum of AGC payment and ESS cost in a power grid with high PV power penetration.

\subsection{Objective function}

When ESS is utilized to control the ramp rate of PV power, the AGC payment including reserve capacity and regulation mileage, of a power grid with high PV penetration will be reduced. Meanwhile, the integration of ESS also incurs investment cost. When ESS has a higher capacity, the PV power can be better smoothed and more AGC payment can be decreased. Hence, there is a tradeoff between the reduction of AGC payment and the ESS investment cost. For this reason, a cost-benefit-based optimization model is built aiming to 
maximize the net benefit from the frequency regulation when ESS is integrated in the grid. Specifically, the objective function is built as follows.

$$
\operatorname{Max} B=\left|C^{\prime} A E-C_{A}\right|-C_{E S S}
$$

where $B$ is the net benefit. $C_{A}$ is the annual cost of frequency regulation considering the AGC reserve capacity and regulation mileage without ESS. $C^{\prime} A E$ is the annual cost of frequency regulation when ESS is integrated. Hence, $\left|C^{\prime} A E-C_{A}\right|$ can show the annual equivalent benefit from frequency regulation when ESS is utilized. $C_{E S S}$ is the annual equivalent investment cost of ESS. It should be pointed out that considering the annual periodic feature of PV power, annual equivalent benefit and cost are calculated. Correspondingly, the objective function aims to maximize the net benefit by achieving the balance between the equivalent benefit incurred by ESS and the ESS investment cost, and the power capacity and energy capacity of ESS are decision variables in the objective function. Subsequently, the calculation of the equivalent benefit and ESS investment cost are detailedly described as follows.

(1) Calculation of the annual equivalent benefit

The annual equivalent benefit is incurred by the reduction of the AGC payment. Twocomponent market-based compensation scheme is applied to determine the AGC payment in this paper, which is issued in Federal Energy Regulatory Commission (FERC) order 755 [18]. The first component is capacity payment depending on AGC reserve capacity which has been discussed in Section 1.1, and the second component is performance-based payment depending on the AGC regulating up/down mileage and performance score of AGC units when responding to AGC regulation commands. In this paper, AGC regulating mileage is calculated according to the CAISO Pay for Performance Regulation (PFPR) document [19]. When AGC units are ordered to respond upwards, if the response power is smaller than the command, AGC regulating mileage payment will be paid according to the actual AGC regulating power; otherwise, it will be paid according to the command value. When AGC units are regulating downwards, inversely, if the response is smaller, it will be paid according to the command; otherwise, it will be paid according to the actual response. Specially, if AGC regulation is opposite to the command, the regulation mileage is set as zero.

Accordingly, the AGC regulating mileage at time $t$ is expressed as follows [19]:

$$
M_{A G C}(t)=\left\{\begin{aligned}
& \max \left(0,\left(\min \left(S_{R E, t}, S_{A G C, t}\right)-S_{R E, t-\Delta t}\right)\right) \\
& \text { if } S_{\mathrm{AGC}, t}>S_{\mathrm{AGC}, t-\Delta t} \\
& \max \left(0,\left(S_{R E, t-\Delta t}-\max \left(S_{R E, t}, S_{A G C, t}\right)\right)\right) \\
& \text { if } S_{\mathrm{AGC}, t}<S_{\mathrm{AGC}, t-\Delta t}
\end{aligned}\right.
$$

where $M_{A G C}(t)$ is the AGC regulation mileage at $t . \Delta t$ is the time interval between the adjacent AGC commands. $S_{R E, t}$ and $S_{R E, t-\Delta t}$ are the response values of AGC units at $t$ and $t$ - 
$\Delta t . S_{A G C, t}$ and $S_{A G C, t-\Delta t}$ are the commands delivered to AGC units at $t$ and $t-\Delta t$. Therefore, the AGC regulation mileage of the $j^{\text {th }}$ hour $M_{A G C}\left(T^{j}\right)$ is expressed as

$$
M_{A G C}\left(T^{j}\right)=\sum_{i=1}^{N} M_{A G C}\left(t_{0}^{j}+i \cdot \Delta t\right)
$$

where $T^{j}$ shows the $j^{\text {th }}$ hour. $N$ is the number of total command intervals during the $j^{\text {th }}$ hour. tj 0 is the initial moment of the $j^{\text {th }}$ hour.

Moreover, the mileage payment is also related to the AGC performance, which is evaluated by measuring how well the AGC units follow the regulation command. Specifically, the first step is getting the absolute deviation $D_{t}$ between the response power of units and the command values, which is expressed as follows.

$$
D_{t}=\left|S_{A G C, t}-S_{R E, t}\right|
$$

In the proposal presented by CASIO, an evaluation index $\gamma_{t}$ is given by a ratio between the deviation $D_{t}$ and the regulation command [19].

$$
\gamma_{t}=\frac{D_{t}}{\left|S_{A G C, t}\right|}=\left|\frac{S_{A G C, t}-S_{R E, t}}{S_{A G C, t}}\right|
$$

When the AGC response power exactly follows the commands, $\gamma_{t}$ will be equal to 0 . The higher $\gamma_{t}$ implies the worse performance of the AGC units. It should be noted that when reverse response occurs, which means the response power $S_{R E, t}$ is opposite to the command value $S_{A G C, t}, \gamma_{t}$ will be higher than 1. Then the score to show the AGC regulation performance $\delta_{\text {score }}(t)$ can be defined as follows [19], [20].

$$
\delta_{\text {score }}(t)=\max \left[0,1-\delta_{t}\right]
$$

where $\delta_{\text {score }}(t)$ is the AGC regulation score.

Subsequently, the performance score of the $j^{\text {th }}$ hour $\delta_{\text {score }}\left(T^{j}\right)$ can be calculated.

$$
\delta_{\text {score }}\left(T^{j}\right)=\frac{\sum_{i=1}^{N} \delta_{\text {score }}\left(t_{0}^{j}+i \cdot \Delta t\right)}{N}
$$

Therefore, the AGC mileage payment for the $j^{\text {th }}$ hour $C_{A G C \text {,mile }}\left(T^{j}\right)$ is expressed as

$$
C_{A G C, \text { mile }}\left(T^{j}\right)=p_{m} \cdot M_{A G C}\left(T^{j}\right) \cdot \delta_{\text {score }}\left(T^{j}\right)
$$

where $p_{m}$ is the price per unit mileage in $\$ / \mathrm{MW}$. The value of $p_{m}$ is determined according to the average market clearing price in CAISO market, e.g., $p_{m}=3.8 \$ / \mathrm{MW}$ [21].

Similarly, the AGC reserve capacity payment for the $j^{\text {th }}$ hour $C_{A G C \text {, cap }}\left(T^{j}\right)$ is expressed as

$$
C_{A G C, \text { cap }}\left(T^{j}\right)=p_{p} \cdot P\left(T^{j}\right)
$$

where $P\left(T^{j}\right)$ is the AGC reserve capacity of the $j^{t h}$ hour, as determined in Section 1.1. $p_{p}$ is the price of AGC reserve capacity per unit size in $\$ / \mathrm{MW}$, and the value of $p_{p}$ is set as 25 \$MW [21]. 
Consequently, the annual AGC regulation payment $C_{A}$ can be determined as the sum of AGC mileage payment and AGC reserve capacity payment.

$$
\begin{aligned}
C_{A} & =\sum_{j=1}^{H r}\left(C_{A G C, \text { mile }}\left(T^{j}\right)+C_{A G C, \text { cap }}\left(T^{j}\right)\right) \\
& =\sum_{j=1}^{H r}\left(p_{m} \cdot M_{A G C}\left(T^{j}\right) \cdot \delta_{\text {score }}\left(T^{j}\right)+p_{p} \cdot P\left(T^{j}\right)\right)
\end{aligned}
$$

where $\mathrm{Hr}$ is the number of hours in a year, i.e. 8760 .

Afterwards, as described in Section 2.2, when ESS is integrated in a power gird with high PV power penetration, the payment of both AGC mileage and AGC reserve capacity will be reduced. Consequently, according to the presented control rules of ESS, when ESS is integrated, the annual AGC regulation payment $C^{\prime} A E$ can also be calculated as follows.

$$
C_{A E}^{\prime}=\sum_{j=1}^{H r}\left(p_{m} \cdot M_{A G C}^{\prime}\left(T^{j}\right) \cdot \delta_{\text {soore }}^{\prime}\left(T^{j}\right)+p_{p} \cdot P^{\prime}\left(T^{j}\right)\right)
$$

where $M_{A G C}^{\prime}\left(T^{j}\right)$ is the AGC regulation mileage of the $j^{\text {th }}$ hour when ESS is integrated. Similarly, $\delta_{\text {score }}^{\prime}\left(T^{j}\right)$ and $P^{\prime}\left(T^{j}\right)$ are the corresponding parameters when ESS is integrated.

(2) Calculation of the annual equivalent investment cost of ESS

The investment cost of ESS mainly includes the fundamental cost and the maintenance cost.

a) Fundamental Cost: The fundamental cost is mainly contributed by the power control system (PCS) and the energy storage pack. Specially, the cost of PCS is incurred by the power inverter and directly related with the rated power capacity of ESS. Meanwhile, the cost of energy storage pack is mainly determined by the rated energy capacity of ESS. Accordingly, the annual fundamental cost of ESS $C_{f u n}$ can be shown as follows.

$$
C_{\text {fun }}=\left(\frac{1}{N_{P}} \cdot \chi \cdot P_{r a t} \cdot \frac{(1+g)^{N_{P}}}{(1+\mathrm{int})^{N_{P}}}+\frac{1}{N_{V}} \cdot \psi \cdot V_{\text {rat }} \cdot \frac{(1+g)^{N_{V}}}{(1+\mathrm{int})^{N_{V}}}\right)
$$

where $\chi$ and $\psi$ are the cost of PCS and energy storage pack per unit size, respectively. $P_{\text {rat }}$ and $V_{r a t}$ are the rated power capacity and energy capacity of ESS, respectively. Here, $C_{f u n}$ takes the time value of capital into consideration [22]. int is the annual interest rate and $g$ is the annual inflation rate. $N_{P}$ and $N_{V}$ are the lifetime of PCS and energy storage pack, respectively. Since nowadays power inverter is a mature technology, $N_{P}$ is determined as 20 years in this paper. In terms of $N_{V}$, the equivalent full cycle method is used for estimating the lifetime of energy storage pack to failure [22]. The equivalent full cycles are defined as the number of cycles to failure multiplied by the depth of discharge (DOD). Afterwards, accordingly to the usage of energy storage pack during the whole year, the lifetime of ESS can be calculated accordingly [11].

b) Maintenance Cost: The maintenance cost generated during the operation of ESS is introduced. Normally, the maintenance cost of ESS can be treated as a fix value, which is $\mu \%$ of the fundamental cost. In this paper, $\mu$ is set as 2 . 
Correspondingly, the annual equivalent ESS investment $\operatorname{cost} C_{E S S}$ can be calculated as follows.

$$
C_{E S S}=(1+\mu) \cdot\left(\frac{1}{N_{P}} \cdot \chi \cdot P_{r a t} \cdot \frac{(1+g)^{N_{P}}}{(1+\mathrm{int})^{N_{P}}}+\frac{1}{N_{V}} \cdot \psi \cdot V_{r a t} \cdot \frac{(1+g)^{N_{V}}}{(1+\mathrm{int})^{N_{V}}}\right)
$$

Based on the proposed cost models, the objective function for ESS sizing can be formulated as,

$$
\begin{aligned}
& \operatorname{Max} . B=\left|\begin{array}{l}
\sum_{j=1}^{H r}\left(p_{m} \cdot M_{A G C}^{\prime}\left(T^{j}\right) \cdot \delta_{\text {seore }}^{\prime}\left(T^{j}\right)+p_{p} \cdot P^{\prime}\left(T^{j}\right)\right) \\
-\sum_{j=1}^{H r}\left(p_{m} \cdot M_{A G C}\left(T^{j}\right) \cdot \delta_{\text {score }}\left(T^{j}\right)+p_{p} \cdot P\left(T^{j}\right)\right)
\end{array}\right|-(1+\mu) . \\
& \left(\frac{1}{N_{P}} \cdot \chi \cdot P_{\text {rat }} \cdot \frac{(1+g)^{N_{P}}}{(1+\mathrm{int})^{N_{P}}}+\frac{1}{N_{V}} \cdot \psi \cdot V_{\text {rat }} \cdot \frac{(1+g)^{N_{V}}}{(1+\text { int })^{N_{V}}}\right)
\end{aligned}
$$

\subsection{Constrains}

Considering the damage incurred by a frequent deep discharge, the SOC of ESS SOC $(t)$ is constrained within a reasonable range.

$$
S O C_{\min } \leq S O C(t) \leq S O C_{\text {max }}
$$

where $\left[S O C_{\min }, S O C_{\max }\right]$ is the range of SOC.

\section{Case study}

To validate the effectiveness of the presented sizing methodology, the simulation based on actual PV power data has been conducted in this paper. Specifically, in a power grid with high PV power penetration, the power generation is mainly composed by thermal

\begin{tabular}{|c|c|c|c|c|}
\hline Type & $\begin{array}{l}\text { Installed capacity per } \\
\text { unit (MW) }\end{array}$ & Number of units & Ramp rate (MW/min) & $\begin{array}{l}\text { Total installed } \\
\text { Capacity (MW) }\end{array}$ \\
\hline TG & 100 & 1 & 6 & 100 \\
\hline TG & 50 & 2 & 3 & 100 \\
\hline TG & 25 & 4 & 1.5 & 100 \\
\hline TG & 20 & 3 & 1 & 60 \\
\hline TG & 15 & 4 & 0.8 & 60 \\
\hline TG & 10 & 8 & 0.5 & 80 \\
\hline PV plant & - & - & - & 31 \\
\hline PV plant & - & - & - & 34 \\
\hline PV plant & - & - & - & 36 \\
\hline PV plant & - & - & - & 24 \\
\hline PV plant & - & - & - & 25 \\
\hline
\end{tabular}
generators and PV power plants, and their installed power capacities are shown in Table I, in which TG means thermal generator.

Table I Installed capacity of each power source in the gird 
The total power capacity of power sources is $500 \mathrm{MW}$, in which the installed capacity of PV power is $150 \mathrm{MW}$. In terms of power load, the typical daily load curves of various seasons are shown in Fig.6, Generally the peak load is about $340 \mathrm{MW}$, while the valley load is about $160 \mathrm{MW}$. For simplicity, it is assumed that there is no power congestion in transmission lines, and the parameters, e.g., active power, reactive power and voltage of each node, are within the reasonable range. Accordingly, the location of power sources and ESS will not affect the ESS size. The typical ESS operation parameters are shown in Table II.
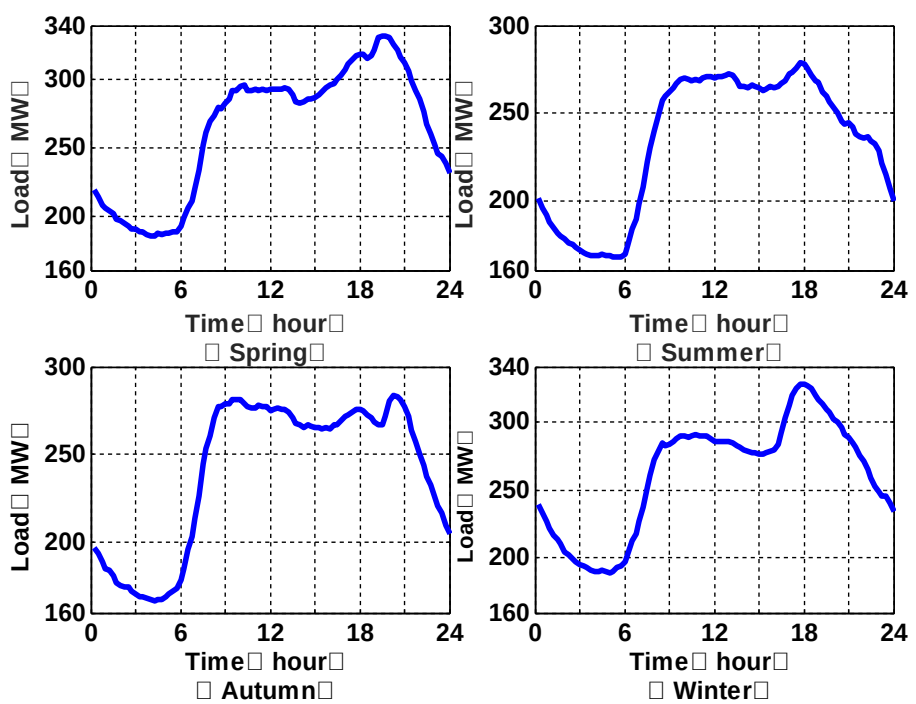

Fig.6 Typical daily load curves of various seasons

Table II Parameter setting

\begin{tabular}{ll||ll}
\hline \hline Parameter & Value & Parameter & Value \\
$\chi(\$ / \mathrm{kW})$ & 700 & $P_{\text {dmax }}(\mathrm{MW})$ & 50 \\
$\psi(\$ / \mathrm{kWh})$ & 350 & SOC $_{\min }$, & 0.2 \\
$p_{p}(\$ \mathrm{MW})$ & 25 & $S O C_{\max }$ & 0.8 \\
$p_{m}(\$ / \mathrm{MW})$ & 3.8 & $g$ & 3 \\
$P_{c \max }(\mathrm{MW})$ & -50 & int & 2 \\
\hline \hline
\end{tabular}

\subsection{Determination of ESS capacity}

Using annual historical PV power data, and according to the presented ESS control and sizing methodology, the energy capacity and power capacity of ESS can be calculated in the simulation.

To demonstrate the relationship between the equivalent benefit and the ESS size, Fig. 7 shows the variation of equivalent benefits along with the ESS power capacity and energy capacity. Specifically, assuming the energy capacity of ESS is fixed, when the power capacity of ESS is increasing, the benefit also increases firstly, and after the peak value point, it begins to decrease. Similarly, assuming the power capacity of ESS is fixed, when the energy capacity of ESS is increasing, the benefit will also increase firstly, and then 
decrease afterwards. Consequently, it can be seen than there will be an optimal ESS size which has the most equivalent benefit. It can be seen that over-sized ESS will not bring more financial return, but seriously decrease the equivalent benefit.

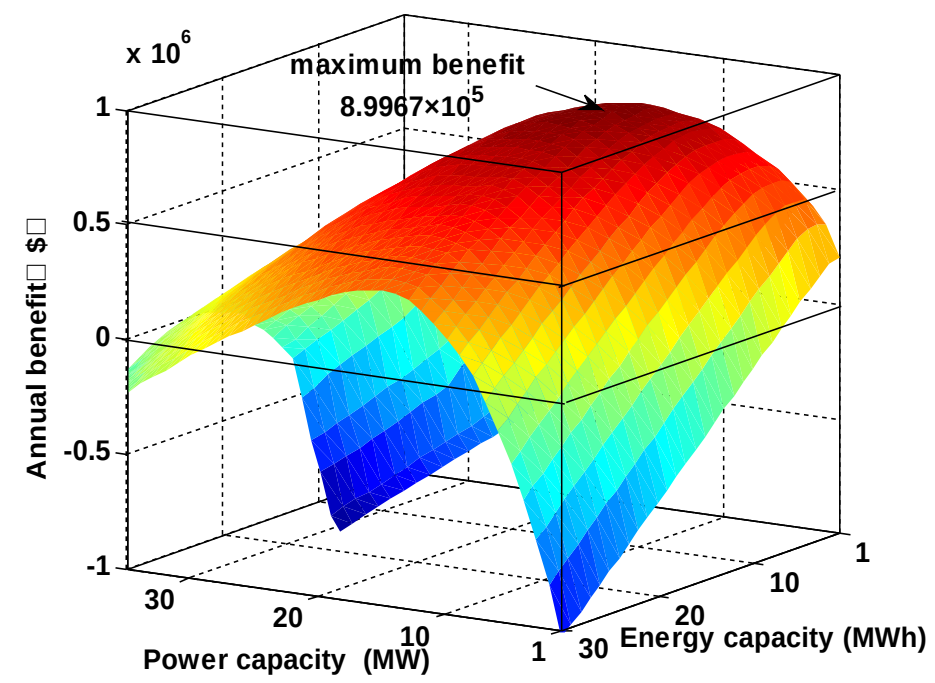

Fig.7 Contour map of equivalent benefits

Using historical PV power data and according to the optimization model, the ESS power capacity and energy capacity are optimally determined, i.e., $12.67 \mathrm{MW}$ and $8.87 \mathrm{MWh}$, respectively. The detailed optimization results are shown in Table III.

Table III optimization results

\begin{tabular}{llll}
\hline \hline Parameter & $C^{\prime} A E(\$)$ & $C_{A}(\$)$ & $V_{\text {rat }}(\mathrm{MWh})$ \\
Value & $1.9760 \times 10^{6}$ & $3.8080 \times 10^{6}$ & 8.87 \\
\hline Parameter & $C_{E S S}(\$)$ & $B(\$)$ & $P_{\text {rat }}(\mathrm{MW})$ \\
Value & $9.3233 \times 10^{5}$ & $8.9967 \times 10^{5}$ & 12.67 \\
\hline \hline
\end{tabular}

From Table III, it can be seen that the annual equivalent ESS cost is 0.93236 million dollars, and from the value difference between $F_{A G C}$ and $F^{\prime}{ }_{A G C}$, the annual reduction of AGC payment can be got, i.e., 1.832 million dollars. Correspondingly, the annual equivalent benefit of ESS can also be calculated, i.e., 0.89964 million dollars.

From the simulation results, it can be seen that the application of ESS in decreasing AGC payment via ramp rate control of PV power has a relatively high return on investment. To further verify the effectiveness of the presented sizing methodology, the economic performance and technical performance of ESS will be demonstrated in the following sections.

\subsection{Economic performance of ESS}

To fully describe the economic performance of ESS, the daily equivalent benefit of ESS has been statistically calculated, as shown in Fig.8. 


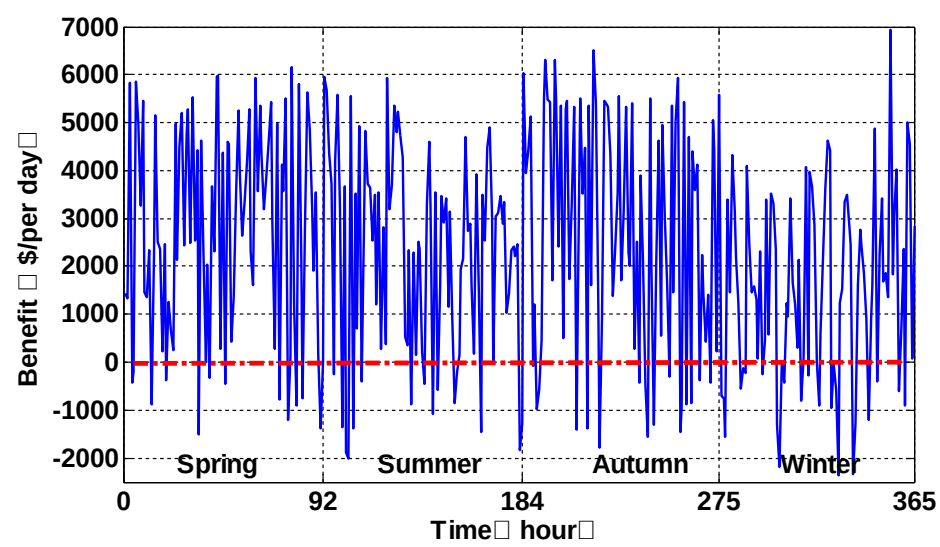

Fig.8 Daily Equivalent benefit of the whole year

From the optimization model of the presented sizing methodology, it should be noted that the more dramatic the PV power fluctuation is, the more reduction of AGC payment can be got. Correspondingly, the more net benefit of ESS can also be achieved. From Fig.8, it can be seen that the benefit has a direct relationship with the weather conditions in various seasons. Specifically, in spring and autumn higher benefit can be got as there are more cloudy and overcast days in these seasons, generating PV power with more dramatic fluctuations. In contrast, in summer, the highest average PV power output appears, but the PV power fluctuation is mild, and then the average benefit is still not high. The same conclusion can be got in winter since there are low average power output and mild fluctuation in this season. From the daily distribution of benefit, it can be seen the highest daily benefit is about 6435 \$, which appears in autumn, while the lowest benefit $-2130 \$$, appearing in the winter. In the days when the PV power is low and the fluctuation is mild, the reduction of AGC payment cannot cover the daily equivalent ESS cost, and then the benefit will be negative. From Fig.8, it can also be statistically calculated that $73.7 \%$ of the days of one whole year have positive benefit, while $27.3 \%$ has negative benefit. Furthermore, the average daily benefit of various weather conditions has also been statistically calculated, and the results show that the average daily benefit are -906 \$, 4732 \$, $3532 \$$ and -789 \$ in sunny, cloud, overcast and rainy days, respectively.

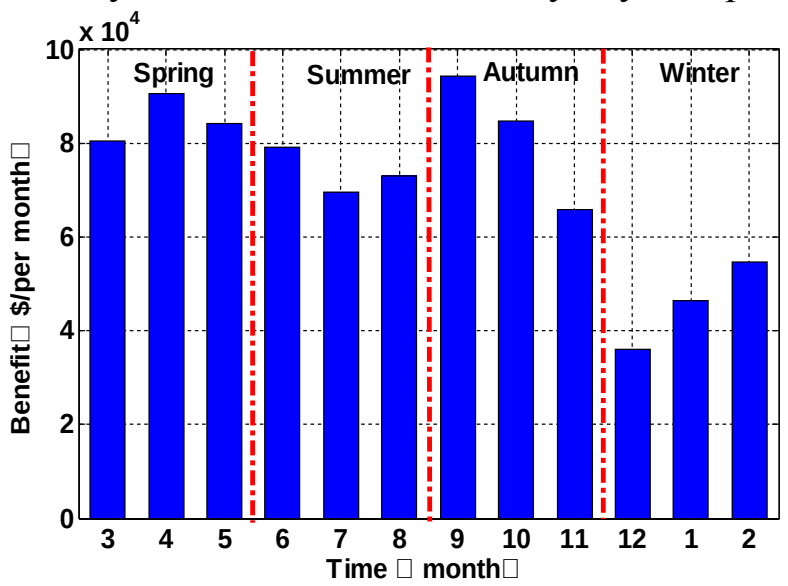

Fig.9 Monthly Equivalent benefit 
Considering the seasonal features of PV power variations, monthly distributions of the equivalent benefit are shown in Fig. 9. Similar conclusion can be got from the monthly benefit curve. Subsequently, the payback period of an ESS $T_{p}$ can also be estimated. $T_{p}$ can be calculated as follows:

$$
T_{p}=\frac{(1+\mu) \cdot\left(\chi \cdot P_{r a t}+\psi \cdot V_{r a t}\right)}{\left|C_{A E}^{\prime}-C_{A E}\right|}
$$

From the cost and benefit analysis above, the payback period of the ESS can be determined as 6.5 years in this paper. Considering the lifetime of PCS and energy storage pack, it can be seen that the application of ESS in a power grid with high PV power penetration has a relatively high return on investment, and a relatively short payback period as well.

From the analysis above, it can be deduced that due to the weather conditions in different geographical areas, the installation of ESS will be more beneficial for the power gird if the PV power shows dramatic fluctuation.

\subsection{Smoothing performance of ESS in the ramp rate control of $P V$ power}

To demonstrate the smoothing performance of ESS in ramp rate control of PV power, the daily equivalent power variation of PV power is presented as an evaluation index to show the smoothing effectiveness.

$$
P_{Y}^{\prime}=\frac{1}{Y} \cdot \sum_{i=1}^{Y} \sum_{t=t_{0}^{\prime}}^{t=t_{v}^{i}}\left|P_{p v}^{\prime}(t)-P_{p v}^{\prime}(t-\Delta t)\right|
$$

where $P^{\prime} Y$ is the equivalent daily power variation of PV power. $Y$ is the days of selected time period. $[t i 0$, ti $y]$ is the time interval of the $i^{\text {th }}$ day. Accordingly, for the purpose of comparison, the daily power variation of PV power without ESS can also be got.

$$
P_{Y}=\frac{1}{Y} \cdot \sum_{i=1}^{Y} \sum_{t=t_{0}^{\prime}}^{t=t_{v}^{i}}\left|P_{p v}(t)-P_{p v}(t-\Delta t)\right|
$$

Hence, the comparison between $P^{\prime} Y$ and $P_{Y}$ shows the smoothing performance of ESS in the ramp rate control of $\mathrm{PV}$ power.

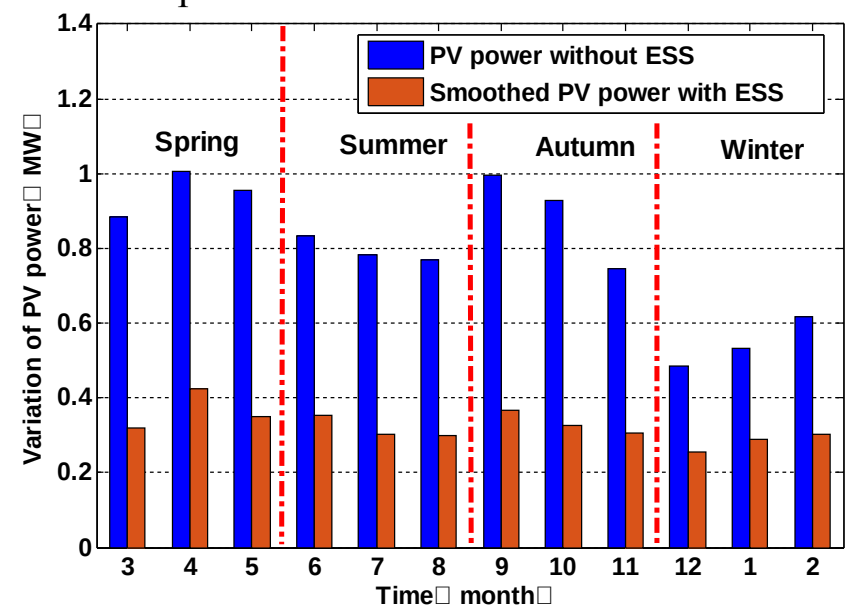

Fig.10 Average monthly power variation of PV power 
According to the calculation of daily power deviation, the average monthly power variation of PV power can also be got, as shown in Fig. 10. From Fig.10, it can be observed that the smoothed PV power with ESS has lower power variation in each month, which means ESS shows an excellent smoothing performance. Furthermore, it can be seen that during the same season, the PV power variation are close, whereas the average value in various seasons are quite different. The reason is that the PV power are seasonal in nature. This can also be concluded from the curve of the smoothed PV power with ESS.

Besides, the daily SOC curves of ESS under typical weather conditions have been chosen to show the operation conditions of ESS, as shown in Fig.11. Correspondingly, the smoothing effectiveness of PV power under various typical weather conditions has also been presented in Fig. 12 to show the performance of ESS.
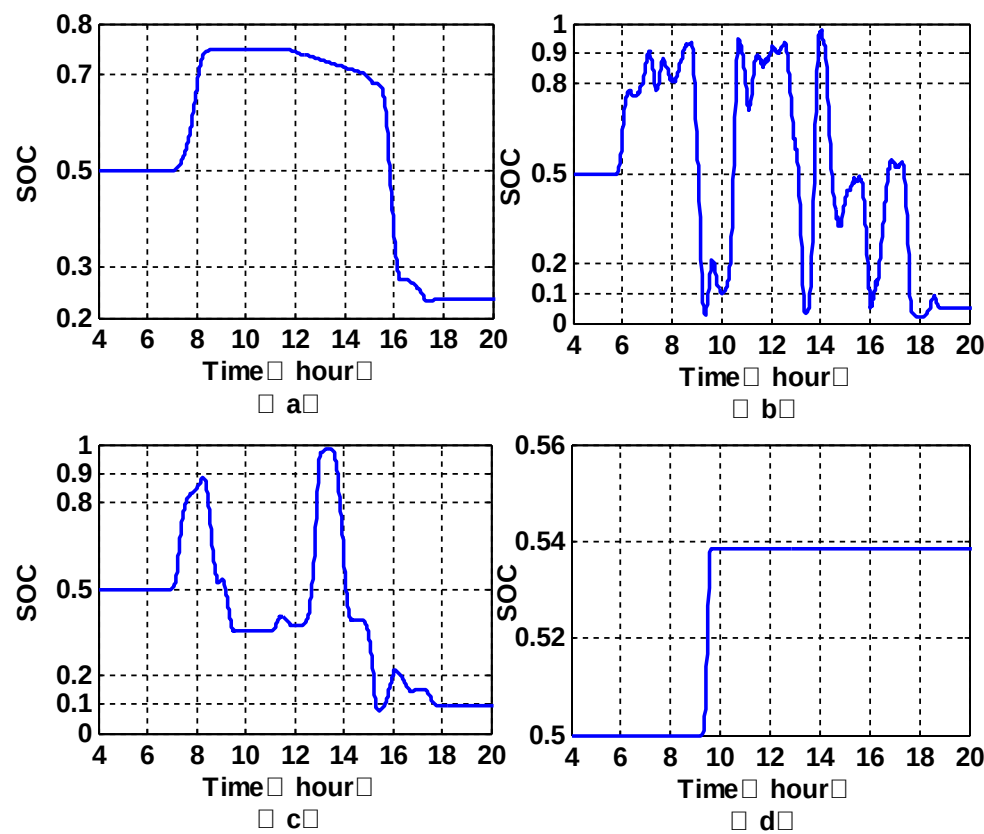

$\square \mathbf{b} \square$

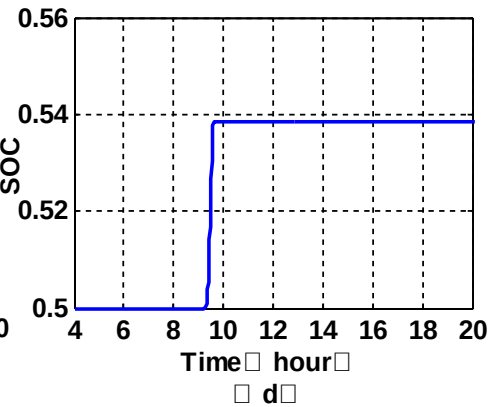

Fig.11 SOC curves under various typical weather

From the Figs. (11) and (12), it can be seen that the weather conditions have a direct relationship with the ESS SOC conditions and the power smoothing effectiveness. Specifically, Fig. 12 (a) shows the typical PV power in sunny days, and there is no obvious fluctuation in PV power. Hence, there is no frequent charge/discharge mode switch of ESS to control the ramp rate of PV power, and accordingly SOC of ESS keeps within a relatively small range, as shown in Fig.11 (a). The PV power of a typical cloudy day is shown in Fig. 12 (b). It can be seen that during [11:00, 16:00], the PV power fluctuates dramatically, and correspondingly the ramp rate of PV power is beyond the limit range. From the charge/discharge curve of ESS, it can be seen that ESS can effectively control the ramp rate of PV power to decrease the power fluctuation. Meanwhile, in Fig.11 (b), the ESS SOC varies within a reasonable range. From the simulation results, it can be seen that 
in cloudy days, ESS plays an important role in dealing with the PV power fluctuations via ramp rate control. Fig. 12 (c) shows the smoothing performance in overcast days, in contrast with cloudy days, the duration of upwards and downward power ramp in overcast days is longer, and the fluctuation of PV power is relatively mild. The reason is that the PV power is mainly related with irradiance and temperature, so when continuously covered by cloud, the PV power will vary along with the change of temperature. Then, the downwards ramp of PV power has a relatively long time duration. Similarly, when the cloud gradually dissipates, the upwards power ramp also shows a long duration due to the increasing temperature. In such a case, the number of charge/discharge intervals of ESS is decreasing, while the charge/discharge power of each interval is increasing. Hence, the SOC of ESS in overcast days still varies in a relatively wide range. Finally, in a rainy day, as shown in Fig. 12 (d), it can be seen that there is no obvious power fluctuation, and the PV power is relatively small. Hence, ESS seldom starts to charge/discharge during most time of this day.
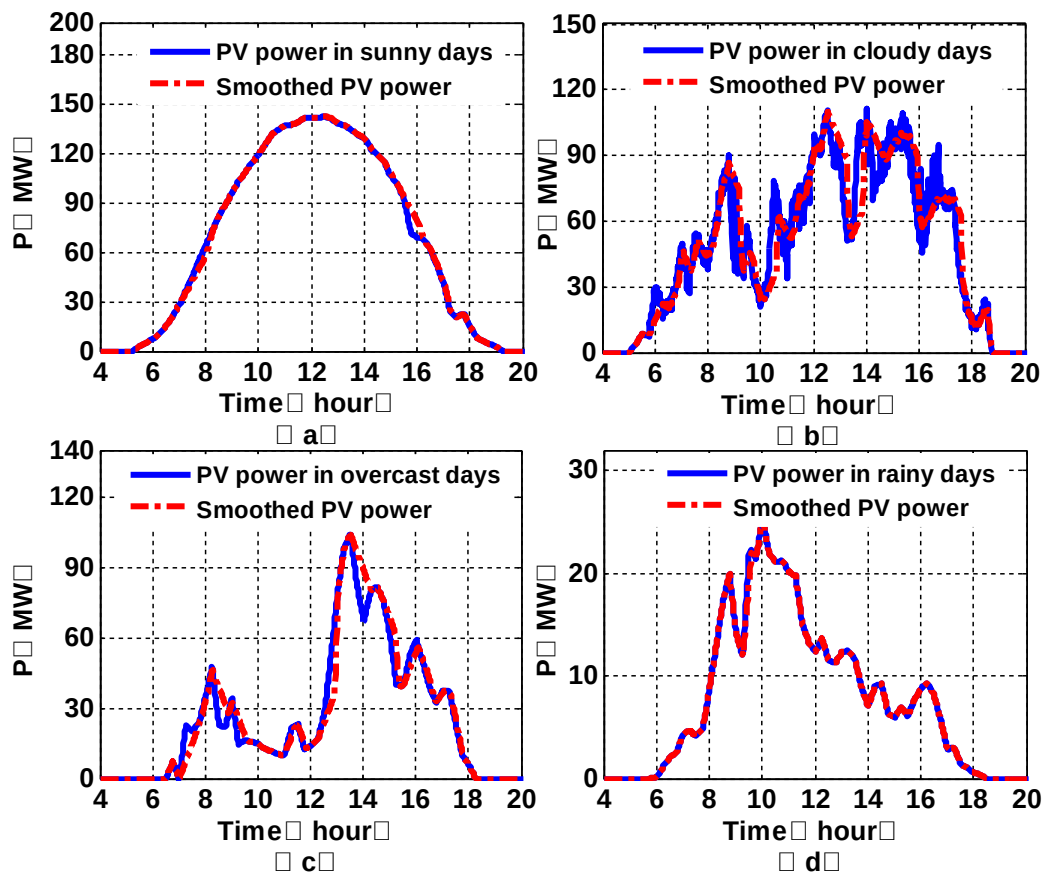

Fig.12 Smoothing performance of ESS under various typical weather

From the analysis of simulation results, it can be seen that the smoothing performance of ESS has been fully demonstrated. ESS can effectively smooth the PV power fluctuation via finitely controlling the ramp rate of PV power. Consequently, the negative impact of PV power fluctuation can be mitigated, and the AGC reserve capacity can also be decreased. Meanwhile, the SOC of ESS keeps operating within a reasonable range.

\section{Conclusion}

A novel ESS sizing methodology in a power grid with high PV power penetration is presented in this paper. Considering the increasing AGC reserve capacity requirement incurred by the rapid fluctuation of PV power, ESS can finitely control the ramp rate of PV 
power to mitigate the fluctuation and correspondingly decrease the total AGC reserve capacity requirement. By maximizing the net benefit incurred by ESS, the presented sizing methodology can achieve the tradeoff between the ESS investment cost and the reduction of AGC payment. Case studies with historical PV power data are fulfilled to demonstrate that the determined ESS can effectively mitigate PV power fluctuation and decrease the AGC payment. Besides, the economic performance and smoothing performance of ESS have been fully demonstrated, and the simulation results show that the ESS integration in a power grid with high PV power penetration is essential, since ESS has a relatively high return on investment and shows satisfactory performance in smoothing PV power fluctuation.

\section{References}

[1] M.J.E.Alam, K.M.Muttaqi, D.Sutanto. A novel approach for ramp-rate control of solar PV using energy storage to mitigate output fluctuations caused by cloud passing. IEEE Trans. Energy Convs. 2014;29(2): 507-517

[2] F. Marra, G. Y. Yang, C. Træholt, J. Østergaard, E. Larsen. Decentralized storage strategy for residential feeders with photovoltaics. IEEE Trans. Smart Grid. 2014;5(2): 974-981

[3] X. R. Xie, Y. H. Guo, B. Wang, Y. P. Dong, et al. Improving AGC performance of coal-fueled thermal generators using multi-MW scale BESS: a practical application. IEEE Trans. Smart Grid.2016; 9(3): 1769-1777

[4] X. Ke, N. Lu, C. Jin. Control and size energy storage systems for managing energy imbalance of variable generation resources. IEEE Trans. Sustain. Energy. 2015; 6(1): 70-78

[5] F. Marra, G. Y. Yang, C. Træholt, J. Østergaard, E. Larsen. Decentralized storage strategy for residential feeders with photovoltaics. IEEE Trans. Smart Grid. 2014; 5(2): 974-981

[6] Wang, Licheng, et al. Real-Time Coordinated Voltage Control of PV Inverters and Energy Storage for Weak Networks with High PV Penetration. IEEE Trans. Power Syst. 2018;33(3):3383-3395

[7] Li, Xiangjun, D. Hui, X. Lai. Battery Energy Storage Station (BESS)-Based Smoothing Control of Photovoltaic (PV) and Wind Power Generation Fluctuations. IEEE Trans. Sustain. Energy. 2013; 4(2): 464-473

[8] Naoto Kakimoto, Hiroyuki Satoh, Satoshi Takayama, et al. Ramp-rate control of photovoltaic generator with electric double-layer capacitor. IEEE Trans. Energy Convs. 2009; 24(2): 465-473

[9] Trudie Wang, Haresh Kamath, Steve Willard. Control and optimization of grid-tied photovoltaic storage systems using model predictive control. IEEE Trans. Smart Grid. 2014; 5(2):1010-1016

[10] Emilio Perez, Hector Beltran, Nestor Aparicio, et al. Predictive power control for PV plants with energy storage. IEEE Trans. Sustain. Energy.2013; 4(2):482-490.

[11] F. Zhang, K. Meng, Z. Xu, et al. Battery ESS planning for wind smoothing via variable-interval reference modulation and self-adaptive SOC control strategy. IEEE Trans. Sustain. Energy. 2017; 8(2):695-707

[12] Bwo-Ren Ke, Te-Tien Ku, Yu-Lung Ke, et al. Sizing the Battery Energy Storage System on a University Campus With Prediction of Load and Photovoltaic Generation. IEEE Trans. Indust. Appl.2016; 52(2): 1136-1147

[13] Yang Ye, Qing Ye, et al. Integrated Size and Energy Management Design of Battery Storage to Enhance Grid Integration of Large-Scale PV Power Plants. IEEE Trans. Industry Electronics. 2017; 65(1): 394402

[14] Yu, Ru, J. Kleissl, S. Martinez. Storage Size Determination for Grid-Connected Photovoltaic Systems. IEEE Trans. Sustain. Energy. 2012; 4(1): 68-81

[15] Zhu, Xiangqi, J. Yan, N. Lu. A Graphical Performance-Based Energy Storage Capacity Sizing Method for High Solar Penetration Residential Feeders. IEEE Trans. Smart Grid. 2016; 8(1): 3-12

[16] Kang, Byung Kwan, et al. A Study on Optimal Sizing of Superconducting Magnetic Energy Storage in Distribution Power System. IEEE Transactions on Applied Superconductivity.2011; 22(3): 3-12 
[17] D. L. Yao, S. S. Choi, K. J. Tseng, T. T. Lie. Determination of short-term power dispatch schedule for a wind farm incorporated with dual-battery energy storage scheme. IEEE Trans. Sustain. Energy. 2012; 3(1): 74-84

[18] FERC order No.755: Frequency regulation compensation in the organized wholesale power markets [EB/OL].[2011-10-06]. https://www.ferc.gov/whats-new/comm-meet/2011/102011/E-28.pdf

[19] CAISO business requirements specification: pay for performance regulation. Document version: 1.4[EB/OL].[2013-05-10]. http://www.caiso.com/Documents/BusinessRequirementsSpecification-PayPerformance.pdf.

[20] Papalexopoulos, Alex D., P. E. Andrianesis. Performance-Based Pricing of Frequency Regulation in Electricity Markets. IEEE Trans. Power Syst. 2013; 29(1):441-449

[21] CAISO ISO market quality and renewable integration: energy imbalance market pricing waiver report February 1 - February 29 2016[EB/OL]. [2016-05-28].http://www.caiso.com/Documents/Mar28_2016_ February2016_EIMPriceWaiverReport_ER15-402.pdf

[22] F. Luo, K. Meng, Z. Y. Dong, Y. Zheng, Y. Chen K. P. Wong. Coordinated operational planning for wind farm with battery energy storage system. IEEE Trans. Sustain. Energy. 2015; 6(1): 253-262 